\title{
Мощные оптоэлектронные коммутаторы нано- и пикосекундного диапазона на основе высоковольтных кремниевых структур с $p-n$-переходами III. Эффекты саморазогрева
}

\author{
(C) A.C. Кюрегян \\ ПАО „НПО Энергомодуль“, \\ 109052 Москва, Россия \\ E-mail: semlab@yandex.ru \\ (Поступила в Редакцию 31 мая 2018 г. \\ В окончательной редакции 24 октября 2018 г. \\ Принята к публикации 29 октября 2018 г.)
}

Впервые теоретически изучены эффекты саморазогрева оптоэлектронных коммутаторов на основе вертикальных высоковольтных структур с $p-n$-переходами (Vertical Photoactivated Semiconductor Switch - VPSS) при работе в высокочастотном режиме. Показано, что сильная зависимость коэффициента поглощения $\kappa(T)$ управляющего излучения от температуры $T$ является основным фактором, определяющим максимальную частоту коммутации $f_{\max }$ и соответствующую максимальную температуру кристалла $T_{\max }$, а также распределения температуры $T$ и плотности тока $j$ по площади VPSS. Двумерный анализ простейшей электротепловой модели VPSS, встроенного в двойную коаксиальную формирующую линию, показал, что увеличение частоты коммутации $f$ приводит к вытеснению тока на периферию прибора, где температура минимальна. Однако при частоте $f<f_{\max }$ и $T<T_{\max }$ распределения $T$ и $j$ по площади прибора остаются устойчивыми. Разумеется, величины $f_{\max }$ и $T_{\max }$ зависят от энергии управляющих импульсов излучения, импульсной коммутируемой мощности и условий теплоотвода. Для VPSS на основе непрямозонных полупроводников $(\mathrm{Si}, \mathrm{SiC})$ они изменяются в пределах 20-120 кГц и $120-160^{\circ} \mathrm{C}$, вполне достаточных для практического применения. Однако VPSS на основе прямозонных полупроводников (GaAs, InP) фактически не пригодны для работы в высокочастотных режимах из-за слишком резкой зависимости $\kappa(T)$.

DOI: 10.21883/FTP.2019.04.47453.8921

\section{1. Введение}

В работах [1-3] были изложены теория и результаты численного моделирования изотермического переключения высоковольтных кремниевых структур с $p-n$-переходами (VPSS - Vertical Photoactivated Semiconductor Switch), управляемых пикосекундными лазерными импульсами, которые позволили получить соотношения между параметрами коммутаторов и характеристиками процесса переключения. Эти результаты применимы для анализа работы и проектирования VPSS на низких частотах $f$, так как за один цикл коммутации в оптимальных режимах рассеивается энергия с

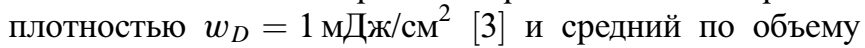
перегрев не превосходит $0.01 \mathrm{~K}$. Однако при $f \geq 50$ кГц VPSS с освещаемой площадью $S_{\mathrm{ph}}=1 \mathrm{~cm}^{2}$ рассеивает среднюю мощность $w_{D} S_{\mathrm{ph}} f \geq 50$ Вт, и его квазистационарный саморазогрев может превышать $100 \mathrm{~K}$, так как в СВЧ тракте (куда должен быть встроен VPSS) трудно обеспечить теплоотвод с сопротивлением $R_{T} \leq 2 \mathrm{~K} / \mathrm{BT}$. Поэтому для решения практических задач проектирования VPSS необходимо ясное понимание эффектов их саморазогрева, которые обладают нетривиальной особенностью.

В обычных высоковольтных приборах повышение температуры $T$ приводит к изменению подвижностей $\mu_{e, h}(T)$ и времен жизни $\tau_{e, h}(T)$ электронов $e$ и дырок $h$, а также к уменьшению контактной разности потенциалов $p-n$-переходов $V_{C}(T)$. Так как обычно длительность формируемого VPSS импульса $t_{R} \ll \tau_{e, h}$ и меньше времени $T_{s c}$ начала возникновения области пространст венного заряда (см. [2]), а падение напряжения на VPSS $U \gg V_{C}$, то последними двумя эффектами можно пренебречь. Но вместо них появляется новый эффект разогрева - увеличение коэффициента поглощения управляющего излучения $\kappa(T)$, анализу которого посвящена настоящая работа. Она является продолжением [2,3], так что все обозначения, конструкция VPSS, режимы освещения и коммутации остались теми же, а все результаты приведены для случая $S_{\mathrm{ph}}=0.5 \mathrm{~cm}^{2}$, площади прибора $S_{D}=2 S_{\mathrm{ph}}$, энергии управляющих импульсов света $W_{\mathrm{ph}}=50$ мкДж, длительности импульсов тока $t_{R}=10$ нс и, если это специально не оговорено, температуры окружающей среды $T_{\text {ext }}=20^{\circ} \mathrm{C}$.

\section{2. Однородный по площади теплоотвод}

Сначала предположим для простоты, что тепло отводится от VPSS однородно по его площади. Суть дела состоит в том, что энергия $W_{D}=w_{D} S_{\mathrm{ph}}$, рассеиваемая прибором за время $t_{R}$, немонотонно зависит от $\kappa$ [3]. При $k d \ll 1$ увеличение $\kappa$ приводит к росту количества порождаемых светом носителей заряда, распределенных почти однородно по толщине кристалла. Поэтому эффективное сопротивление VPSS в проводящем состоянии и, следовательно, $W_{D}$ уменьшаются. При $k d>1$ увеличе- 


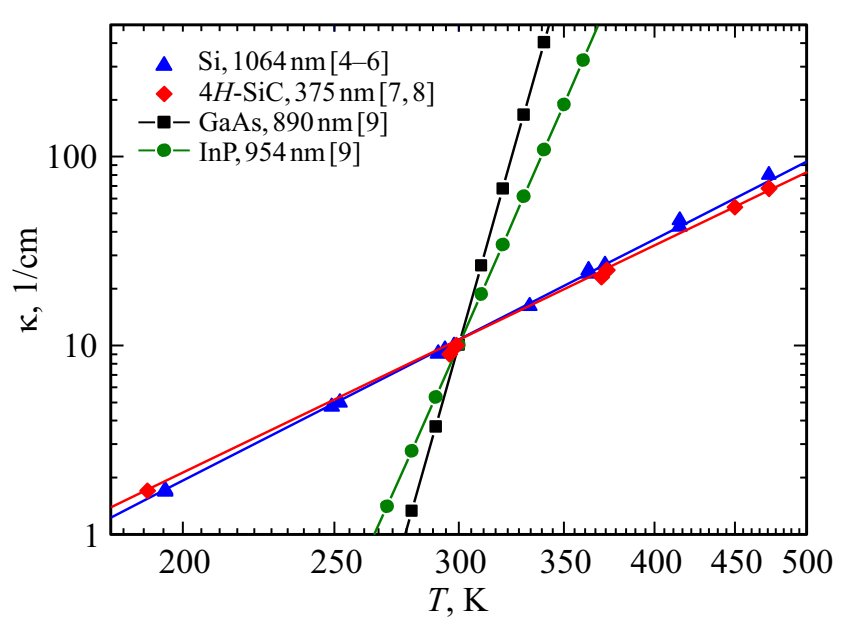

Рис. 1. Температурные зависимости коэффициента поглощения в $\mathrm{Si}$ (треугольники, $\lambda=1064$ нм [4-6]), 4H-SiC (ромбы, $\lambda=375$ нм [7,8]), GaAs (квадраты, $\lambda=890$ нм [9]) и InP (кружки, $\lambda=954$ нм [9]) излучения с длинами волны $\lambda$, для которых $\kappa(300 \mathrm{~K}) \approx 10 \mathrm{~cm}^{-1}$. Линии - аппроксимации по формуле $(1)$ с показателями степени $\gamma=4.25$ для $\mathrm{Si}, \gamma=4.0$ для $4 H-\mathrm{SiC}$, $\gamma=29.0$ для GaAs и $\gamma=19.0$ для InP.

ние $\kappa$ приводит ко все более медленному росту количества носителей заряда, но теперь они сосредоточены в слое с уменьшающейся толщиной $\kappa^{-1}<d$ вблизи освещаемой поверхности, поэтому сопротивление VPSS и $W_{D}$ возрастают при увеличении $\kappa$. Минимум функции $W_{D}(\kappa)$ достигается при $\kappa \approx 2 / d$ (см. рис. 8 в [3]), так что оптимальная длина волны света $\lambda$ попадает на край основной полосы поглощения, где $\kappa$ очень сильно зависит и от $\lambda$, и от $T$. Примеры зависимостей $\kappa(T)$ для четырех полупроводников по данным работ [4-9] приведены на рис. 1. Все они хорошо аппроксимируются степенной функцией

$$
\kappa(T)=\kappa_{0}\left(T / T_{0}\right)^{\gamma},
$$

предложенной авторами работы [5] для поглощения света с $\lambda=1064$ нм в $\mathrm{Si}$. C ростом $\lambda$ показатели степени $\gamma$ слабо увеличиваются, но почти пятикратное различие между их значениями для прямозонных и непрямозонных материалов сохраняется.

Вследствие этого зависимость $W_{D}(T)$ также оказывается немонотонной. Пример такой зависимости для случая однородных распределений тока и температуры по площади кремниевого VPSS, освещаемого светом с $\lambda=1064$ нм, приведен на рис. 2, кривая $A$. Следует отметить, что температурная зависимость подвижностей очень слабо влияет на вид функции $W_{D}(T)$. На это указывают результаты расчетов (кривая $B$ на рис. 2) при различных температурах с учетом реальной зависимости $\mu_{e, h}(T)$ и постоянном значении $\kappa=35 \mathrm{~cm}^{-1}$, соответствующем температуре $100^{\circ} \mathrm{C}$.

При работе в высокочастотном режиме температура очень слабо изменяется за время $1 / f$, по толщине $d$ кристалла и при изменении радиальной координаты $\rho$ на размер элементарной ячейки VPSS, который должен быть меньше или порядка $d$. Поэтому для анализа эффектов саморазогрева приборов с площадью $S_{D} \gg d^{2}$ целесообразно использовать температуру, усредненную по времени $1 / f$ и объему $d^{3}$. Далее для упрощения формул мы будем обозначать эту „среднюю“ температуру тем же символом $T$. После включения прибора $T$ увеличивается с постоянной времени $\tau_{D} \gg 1 / f$ до тех пор, пока средняя по времени рассеиваемая мощность $P_{D}=W_{D}(T) f$ не сравняется с мощностью $\left(T-T_{\text {ext }}\right) / R_{T}$, отводимой системой охлаждения однородно по площади. Через время $t \gg \tau_{D}$ возникает стационарное состояние с температурой прибора $T_{s t}$, которая является минимальным корнем уравнения

$$
T_{s t}=T_{\mathrm{ext}}+W_{D}\left(T_{s t}\right) R_{T} f
$$

где $T_{\mathrm{ext}}$ - температура окружающей среды. Из рис. 2 следует, что его решение существует только при частоте, меньшей некоторого значения $f_{\max }$, которое можно вычислить приближенно по формуле

$$
f_{\max } \approx \frac{T_{\max }-T_{\mathrm{ext}}}{W_{D}\left(T_{\max }\right) R_{T}},
$$

где $T_{\max } \approx T_{W}$, а $T_{W}-$ температура, при которой энергия $W_{D}$ минимальна. В рассматриваемом конкретном случае $T_{\max }=120^{\circ} \mathrm{C}, W_{D}\left(T_{\max }\right) \approx 0.5$ мДж и $f_{\max } \approx 80$ кГц для кремниевого VPSS. Для приближенного вычисления температуры $T_{\max }$ и энергии $W_{D}\left(T_{\max }\right)$, которые зависят от параметров прибора и управляющего импульса света, можно использовать следующее соотношение между плотностью импульса тока $j$ в освещаемых областях и падением напряжения $U_{o n}$ на приборе в

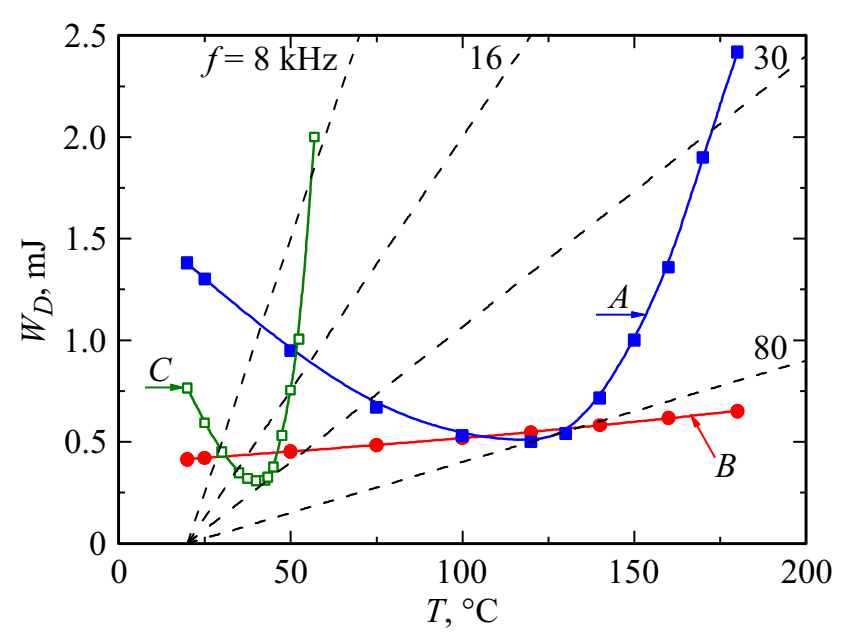

Рис. 2. Сплошные линии - зависимости энергии $W_{D}$ от температуры (см. пояснения в тексте). Штриховые линии энергия $W_{T}=\left(T-T_{\mathrm{ext}}\right) / R_{T} f$, отводимая за время $1 / f$ системой охлаждения с тепловым сопротивлением $R_{T}=2.5 \mathrm{~K} / \mathrm{BT}$ при различной частоте повторения импульсов $f$. Символы результаты численного моделирования в одномерном приближении к данным работы [3]. 


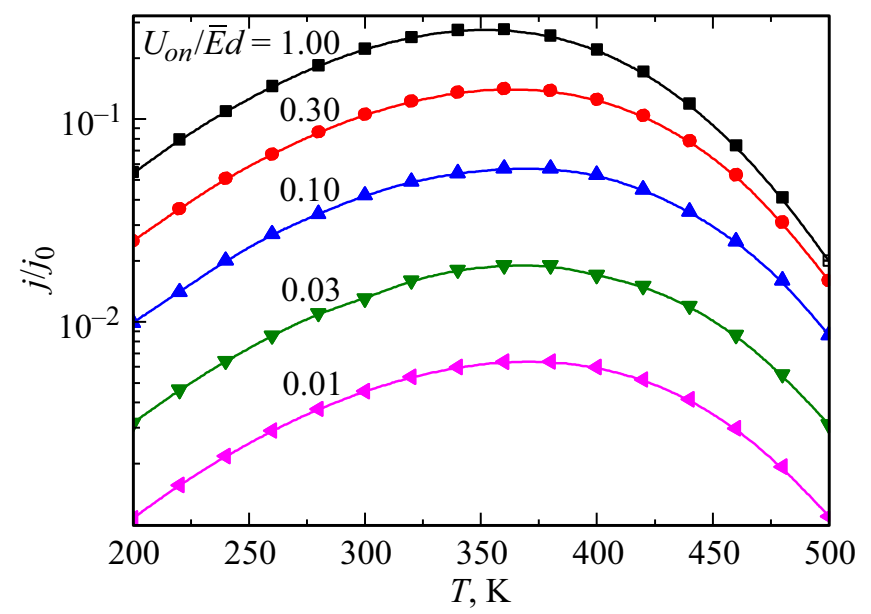

Рис. 3. Зависимости $j\left(T, U_{\text {on }}\right)$, рассчитанные по формуле (4) для „кремниевой“ зависимости $\kappa(T)$.

проводящем состоянии:

$$
\begin{aligned}
j\left(T, U_{\text {on }}\right) & \approx j_{0} \Psi\left[\kappa(T) d, U_{\text {on }} / \bar{E} d\right], \\
\Psi(k, u) & =k \frac{\exp (k u)-1}{\exp [k(u+1)]-1},
\end{aligned}
$$

где $j_{0}=\frac{W_{\mathrm{ph}}}{h \omega} \frac{2 q \bar{v}}{S_{D} d}, h \omega-$ энергия квантов света, $q-$ элементарный заряд. При выводе (4), как и аналогичной формулы (7) из работы [2], мы предполагали выполнение неравенства $t_{R}<t_{s c}$, пренебрегали током смещения в проводящем состоянии прибора и резистивным падением напряжения вдоль электродов, обусловленным растеканием тока. Кроме этого, не учитывались отражение света от тыльного контакта и различие подвижностей электронов и дырок. Эти два упрощения приводят к некоторой количественной погрешности, но зато позволяют на качественном уровне учесть эффект насыщения зависимости дрейфовых скоростей электронов и дырок от напряженности поля $E$ в соответствии с обычной формулой $v(E)=\bar{v} E(E+\bar{E})$, который становится важным при очень малых и очень больших значениях $k d$. Графики зависимостей $j\left(T, U_{o n}\right)$, приведены на рис. 3.

Энергия, рассеиваемая прибором за один импульс длительностью $t_{R}$, равна

$$
W_{D}=W_{C}+t_{R} I U_{o n},
$$

где $W_{C}=C_{D} U_{0}^{2} / 2 \approx 0.24$ мДж - энергия, накопленная в барьерной емкости прибора $C_{D}$ и рассеиваемая им в процессе переключения [3], I - полный ток через прибор, равный

$$
I=\left(U_{0}-U_{o n}\right) / R
$$

$R=2 Z$ для одинарной и $R=Z$ для двойной формирующей линии с волновым сопротивлением $Z$, согласованной с активной нагрузкой. Далее, как и в $[2,3]$, мы будем считать, что $R=10$ Ом. Если температура и ток однородны по площади VPSS, то $I=S_{\mathrm{ph}} j\left(T, U_{\text {on }}\right)$ и совместное использование (4)-(6) позволяет просто найти $T_{\max }$ и $W_{D}\left(T_{\max }\right)$ и вычислить $f_{\max }$ по формуле $(3)$.

\section{3. Радиальный теплоотвод}

Рассмотрение в предыдущем разделе идеализированного случая просняет особенность саморазогрева VPSS, однако на самом деле однородный по площади теплоотвод фактически невозможно реализовать. Дело в том, что высокая скорость переключения может быть „утилизирована“ надлежащим образом, если только сам VPSS является составной частью формирующей линии. Возможный вариант такой конструкции на основе двойной коаксиальной формирующей линии изображен на рис. 4. Тепло отводится от VPSS к радиатору через полупрозрачный молибденовый электрод, обеспечивающий квазиоднородную засветку, и кольцевой керамический изолятор. Радиатор обеспечивает теплоотвод с тепловым сопротивлением $r_{T}$ от внешней образующей поверхности керамического изолятора к окружающей среде. Теплоотвод через дисковую пружину, соединяющую VPSS с внутренним проводником формирующей линии, пренебрежимо мал. Ясно, что в этом случае температура кристалла и, значит, плотность тока не могут быть однородными по площади.

Для вычисления распределений $T(\rho)$ и $j(\rho)$ по радиуcy VPSS необходимо решить уравнение теплопроводно-
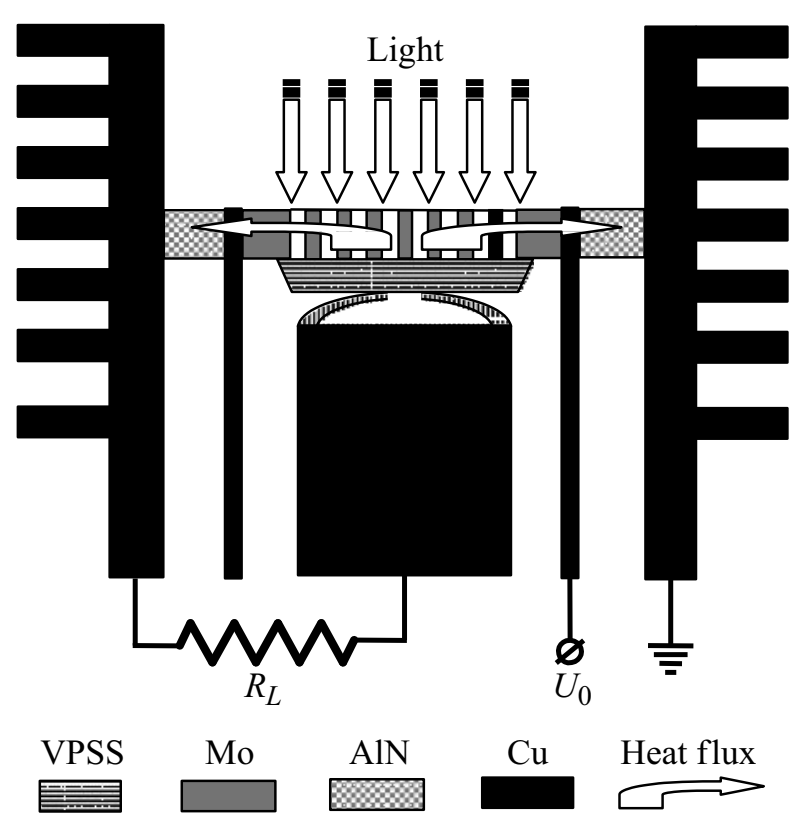

Рис. 4. Схематичное изображение оптоэлектронного коммутатора, присоединенного к торцу двойной коаксиальной формирующей линии с волновым сопротивлением $Z$, согласованной с активной нагрузкой $R_{L}=2 Z[10]$. 

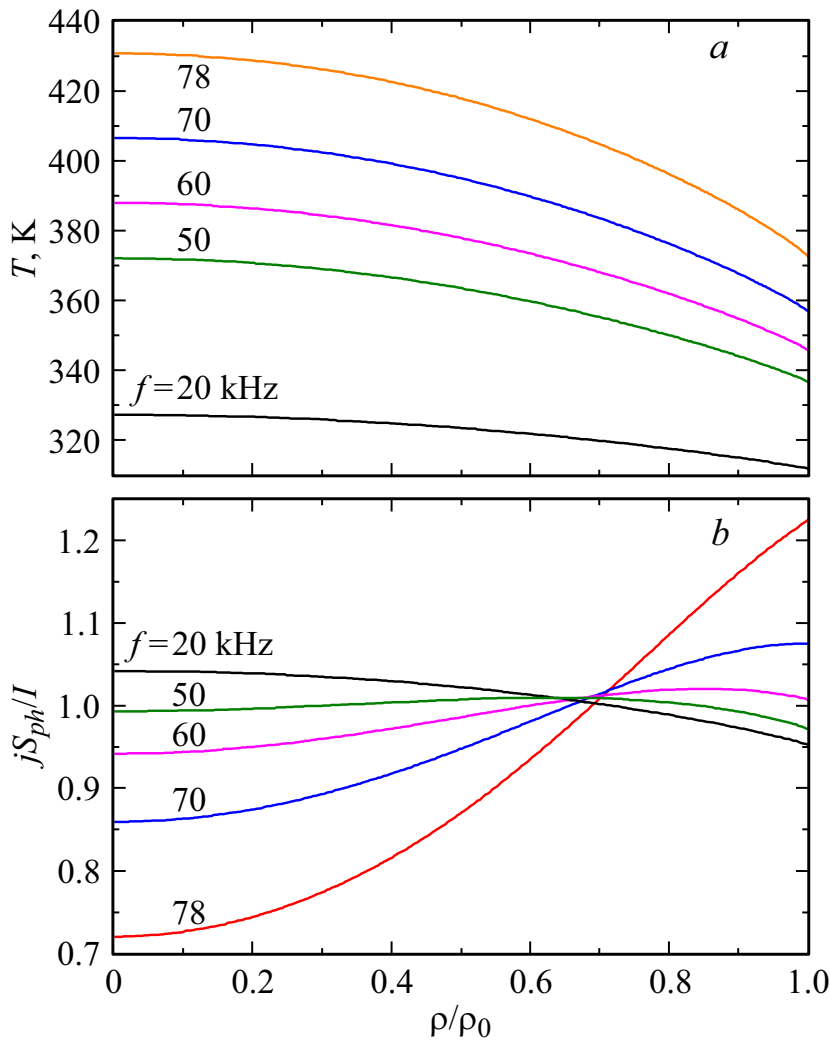

Рис. 5. Радиальные распределения температуры кристалла $(a)$ и нормированной плотности тока $(b)$ при $r_{T}=1 \mathrm{~K} / \mathrm{BT}_{\text {и }}$ различных частотах повторения импульсов $f$.

сти с учетом того, что средняя ${ }^{1}$ плотность мощности $p_{D}$, рассеиваемой пробором, зависит от температуры:

$$
p_{d}\left(T, U_{o n}\right) \approx\left\{W_{C}+t_{R} j_{0} S_{\mathrm{ph}} U_{o n} \Psi\left[\kappa(T) d, U_{o n} / \bar{E} d\right]\right\} f / S_{D},
$$

а полный ток определяется формулой

$$
I=\frac{S_{\mathrm{ph}}}{S_{D}} \int_{S_{D}} j\left(T, U_{o n}\right) d S .
$$

Мы решали эту задачу численно для конструкции, изображенной на рис. 4, используя соотношения (4) -(8) и считая, что радиус молибденового электрода $\rho_{\mathrm{Mo}}=7 \mathrm{Mм}$, внешний радиус керамического изолятоpa $\rho_{\mathrm{AIN}}=9 \mathrm{MM}$, а толщина этих двух деталей равна 1 мм. Результаты расчетов приведены на рис. 5-7.

Как и следовало ожидать, температура в центре прибора $T(0)$ всегда максимальна (рис. 5,a). При малых частотах плотность тока также максимальна в центре и очень слабо уменьшается к периферии прибора. Однако при $f>50$ кГц температура $T(0)$ превышает значение $375 \mathrm{~K}$, при котором достигается максимум функции $j(T)$ (см. рис. 3), тогда как температура на периферии $T\left(\rho_{D}\right)$ остается меньше $375 \mathrm{~K}$. Вследствие

\footnotetext{
1 Усреднение плотности рассеиваемой мощности по времени и координате производилась так же, как усреднение температуры.
}

этого ток вытесняется на периферию прибора (рис. 5, $b$ ), где величина коэффициента поглощения ближе всего к оптимальному значению $\kappa(T) \approx 2 / d$, условия теплоотвода улучшаются и поэтому эффективное тепловое

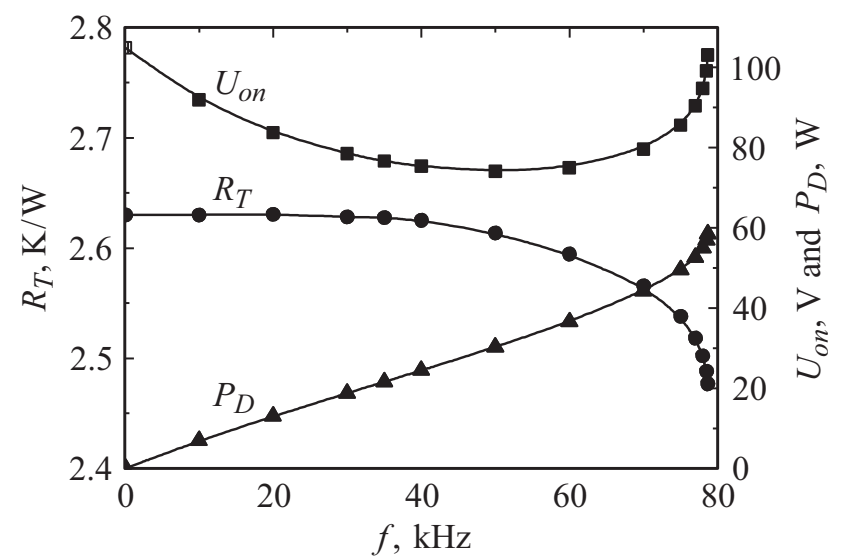

Рис. 6. Зависимости эффективного теплового сопротивления $R_{T}$ (кружки), падения напряжения на VPSS $U_{o n}$ (квадраты) и средней рассеиваемой мощности $P_{D}$ (треугольники) от частоты при $r_{T}=1 \mathrm{~K} / \mathrm{BT}$.

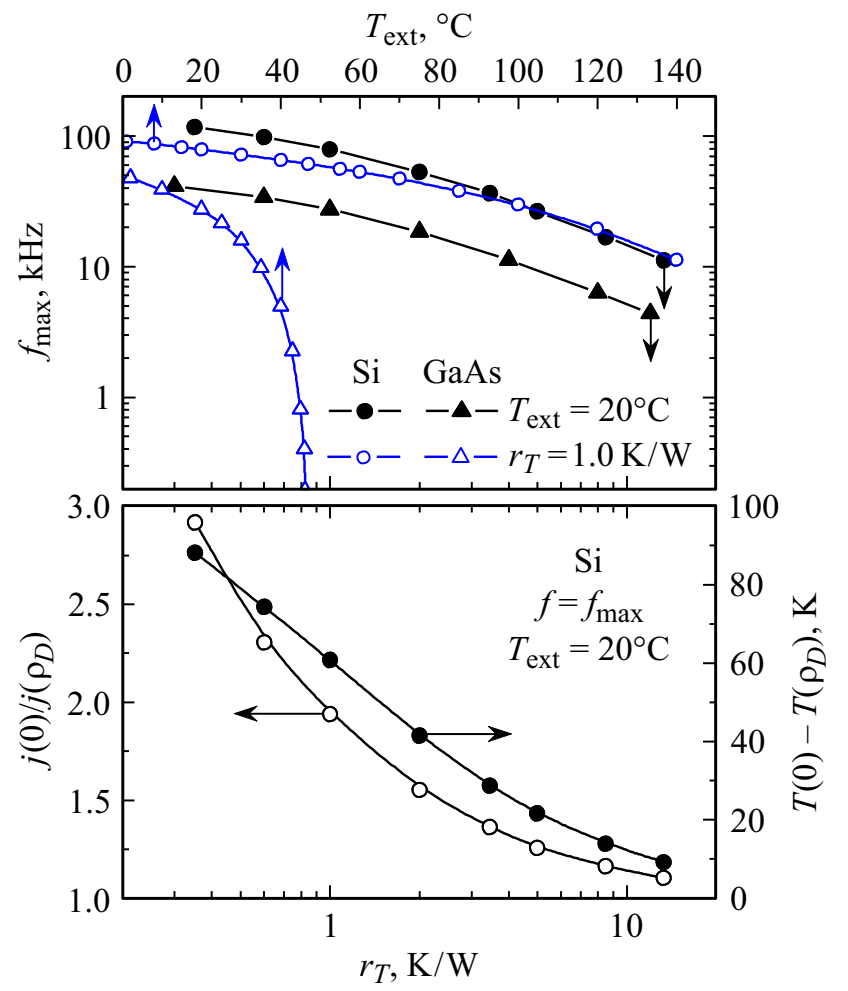

Рис. 7. Вверху - зависимости максимальной частоты повторения имппульсов $f_{\max }$ от $r_{T}$ при $T_{\text {ext }}=20^{\circ} \mathrm{C}$ (темные символы) и от $T_{\mathrm{ext}}$ при $r_{T}=1 \mathrm{~K} / \mathrm{B}$ (светлые символы) для кремниевого (кружки) и арсенид-галлиевого (треугольники) VPSS. Внизу - зависимости отношения плотностей тока (светлые символы) и разности температур (темные символы) в центре и на периферии кремниевого VPSS от $r_{T}$ при $f=f_{\max }$ и $T_{\mathrm{ext}}=20^{\circ} \mathrm{C}$. 
сопротивление $R_{T}=\left[T(0)-T_{\text {ext }}\right] / P_{D}$ уменьшается, как это изображено на рис. 6. Кроме того, при $f>50$ кГц падающая зависимость $U_{o n}(f)$ сменяется на быстро нарастающую. Это приводит к сверхлинейному росту рассеиваемой мощности $P_{D}(f)=U_{o n} I$, так как при нормальной работе VPSS $U_{\text {on }} \ll U_{0}$ и полный ток $I \approx U_{0} / R$, т.е. практически не зависит от $f$ (см. формулу (6)). С ростом $f$ мощность $P_{D}$ нарастает быстрее, чем падает тепловое сопротивление $R_{T}$ (см. рис. 6), поэтому при $f>f_{\text {max }}$ условия существования стационарного состояния перестают выполняться и наступает тепловой пробой.

\section{4. Заключение}

В заключение рассмотрим зависимость $f_{\text {max }}$ от параметров VPSS, управляющих импульсов света, формирующей линии и условий теплоотвода. В качестве примера на рис. 7 приведены зависимости $f_{\max }$ от $r_{T}$ и $T_{\text {ext. }}$ Как и следовало ожидать, $f \max$ увеличивается при уменьшении $r_{T}$ и $T_{\text {ext }}$, но распределения температуры $T(\rho)$ и плотности тока $j(\rho)$ по радиусу $\rho$ кристалла становятся все более неоднородными. Однако эти распределения остаются устойчивыми вследствие того, что при $T>T_{W}$ плотность тока уменьшается с ростом температуры кристалла.

Видно также, что кремниевые VPSS могут работать в высокочастотном режиме при очень высоких температурах окружающей среды. Это качество, обусловленное тем, что зависимость $\kappa(T)$ в $\mathrm{Si}$ не слишком резкая $(\gamma=4.25)$, присуще также и VPSS на основе карбида кремния $(\gamma=4.0)$. В этом отношении VPSS на основе прямозонных полупроводников типа $\mathrm{GaAs}$ и InP значительно хуже. Во-первых, и при $T_{\text {ext }}=20^{\circ} \mathrm{C}$ их максимальная рабочая частота оказывается в $\sim 3$ раза меньше, несмотря на то что суммарная подвижность электронов и дырок в GaAs в 4 раза больше, чем в $\mathrm{Si}$. Во-вторых, $f_{\text {max }} \rightarrow 0$ при $T_{\text {ext }} \rightarrow 45^{\circ} \mathrm{C}$, так что при бо́льших температурах арсенид-галлиевые VPSS вообще не могут функционировать в частотном режиме. Причиной этого является гораздо бо́льшее значение $\gamma$ (см. рис. 1) и соответствующее очень резкое возрастание $W_{D}(T)$ при отклонении температуры кристалла от значения $T_{W} \approx 40^{\circ} \mathrm{C}$, изображенное кривой $C$ на рис. 2 .

Работа выполнена при поддержке РФФИ (грант № 16-08-01292).

\section{Список литературы}

[1] А.С. Кюрегян. ФТП, 48, 1686 (2014).

[2] А.С. Кюрегян. ФТП, 51, 1257 (2017).

[3] А.С. Кюрегян. ФТП, 51, 1263 (2017).

[4] G.G. Macfarlane, T.P. McLean, J.E. Quarrington, V. Roberts. Phys. Rev., 111, 1245 (1958).

[5] K.G. Svantesson, N.G. Nilsson. J. Phys. C: Sol. St. Phys., 12, 3837 (1979).
[6] H.A. Weakliem, D. Redfield. J. Appl. Phys., 50, 1491 (1979).

[7] P. Grivickas, V. Grivickas, J. Linnros, A. Galeckas. J. Appl. Phys., 101, 123521 (2007).

[8] N. Watanabe, T. Kimoto, J. Suda. Jpn. J. Appl. Phys., 53, 108003 (2014).

[9] M. Beaudoin, A.J.G. DeVries, S.R. Johnson, H. Laman, T. Tiedje, Appl. Phys. Lett., 70 (26), 3540 (1997).

[10] C.S. Chang, V.K. Mathur, M.J. Rhee, C.H. Lee, Appl. Phys. Lett., 41 (5), 392 (1982).

Редактор Г.А. Оганесян

\section{High-power nano- and picosecond optoelectronic switches based on high-voltage silicon structures with $p-n$-junctions III. Self-heating effects}

\section{A.S. Kyuregyan \\ NPO Energomodule, 109052 Moscow, Russia}

Abstract Self-heating effects of picosecond optoelectronic switches based on vertical high-voltage structures with $p-n$-junctions (VPSS) operating in high-frequency mode were theoretically studied for the first time. It is shown that strong temperature dependence of the control radiation absorption coefficient $\kappa(T)$ is the main factor determining the maximum switching frequency $f_{\max }$ and the corresponding maximum crystal temperature $T_{\max }$, as well as distributions of temperature $T$ and current density $j$ over a device area. A two-dimensional analysis of the simplest electrothermal model of VPSS embedded into a double coaxial forming line showed that an increase in the switching frequency $f$ leads to displacement of current to device periphery, where the temperature is minimal. However, distributions of $T$ and $j$ over the device area remain stable if $f<f_{\max }$ and $T<T_{\max }$. Of course, the values $f_{\max }$ and $T_{\max }$ depend on the energy of control radiation pulses, pulse switching power and heat sink conditions. For VPSS based on nondirectgap semiconductors $(\mathrm{Si}, \mathrm{SiC})$, they varies within $20-120 \mathrm{kHz}$ and $120-160^{\circ} \mathrm{C}$, quite sufficien for practical application. However, VPSS based on direct-gap semiconductors (GaAs, InP) are not actually suitable for operation in high-frequency modes due to too sharp dependence $k(T)$. 